\title{
Cambios en las Relaciones y Satisfacciones Intergeneracionales Asociados al Uso de las TICs
}

\section{Intergenerational Relations Changes and Satisfaction Associated to the ICTs Use}

\author{
Cristina Figuer Ramírez, Sara Malo Cerrato e Irma Bertran Camats \\ Universitat de Girona
}

\begin{abstract}
Resumen. En el presente artículo se exponen los datos obtenidos de dos muestras de adolescentes catalanes de entre 12 y 16 años y de sus progenitores en 2003 y en 2008 . El objetivo es explorar los cambios en las relaciones entre progenitores e hijos/as y la incidencia que el uso de las TICs tiene en estos cambios. Los resultados muestran que con el paso del tiempo se da una mayor implicación de los progenitores en la comunicación con sus hijos/as acerca de este tema, cosa que no conlleva una mayor satisfacción de los/las adolescentes con dicha comunicación posiblemente por la percepción de falta de dominio de su padre o madre.

Palabras clave: tecnologías, adolescentes, relaciones intergeneracionales, comunicación, satisfacción.
\end{abstract}

\begin{abstract}
This article presents data from two samples of Catalan adolescents from 12 to 16 years and their parents in 2003 and 2008. The main aim is to explore the changing relationships between parents and their children and the impact that the use of ICT has on these changes. The results show that over the time there is a greater involvement of parents in communicating with their children about this issue, which involves no greater satisfaction of adolescents with such communication, possibly by the perception of lack of control that his/her father or mother has.

Key words: Technologies, adolescents, intergenerational relationships, communication, satisfaction.
\end{abstract}

\section{Introducción}

Las dinámicas sociales actuales y los acelerados avances tecnológicos están produciendo importantes cambios en las interacciones entre los adultos y los/las adolescentes (Alsinet, Pérez y Agulló, 2003). Como más relevantes podemos destacar: (a) la progresiva infantilización que repercute en las formas de aspirar a la adolescencia, donde se producen importantes transformaciones y dificultades para acceder al mundo de los adultos y (b) los cambios en

La Correspondencia sobre este artículo debe enviarse a la segunda autora a la Universidad de Girona, Facultad de Educación y Psicología, Departamento de Psicología (Despacho 106), Plaza Sant Domènec, 9. 17071 Girona. E-mail: sara.malo@udg.edu las formas de entender e interpretar los nuevos valores sociales y familiares (Casas, Figuer et al., 2005; Casas, González et al., 2007).

La aparición de las TICs parece ser que está incrementando la diferenciación categorial entre el grupo de los/las adolescentes y el grupo de los adultos. Para los chicos/as, el hecho de disponer y usar determinadas tecnologías es un símbolo de identidad que les otorga un cierto estatus y que, por tanto, les facilita crear barreras imaginarias entre su mundo y el de los adultos. Contrariamente, para los adultos el hecho de que un hijo/a disponga de determinadas tecnologías, como el teléfono móvil, les puede servir para incrementar la percepción de seguridad del hijo/a (y la propia) y para tener la sensación de 
mayor control de lo que estos hacen (Malo, 2009). Como apunta Casas $(1998,2006)$, los adultos de nuestro entorno socio-cultural tenemos el reto de intentar comprender por qué hemos estado interesados en mantener a los niños/as y adolescentes en la categoría homogénea y separada de los "menores", en lugar de profundizar en los procesos de socialización y en la construcción de nuevos consensos sociales con la nuevas generaciones.

Parece evidente que la aparición de las TICs está creando lo que algunos autores denominan brecha generacional (Feixa, 2001). Los/las adolescentes, y cada vez más los niños y niñas son expertos manipuladores de los medios electrónicos, de manera que la mayoría de los adultos pasan a un segundo plano en términos de socialización en este aspecto. En este sentido, Harris $(1995,2006)$ apunta, en su teoría de la socialización grupal (Group Socialization Theory), que los procesos de socialización de los/las niños/as y los/las adolescentes se producen con el grupo de iguales, fuera del entorno familiar. El lugar de los progenitores es ocupado por el del grupo de iguales que son con los que aprenden a utilizar estos medios.

Tradicionalmente se había concebido a la familia como el primer y básico agente socializador. Ya hace décadas que se plantea la importancia de articular el binomio familia-escuela, por su transcendental influencia en el proceso de desarrollo y socialización infantil. En el último cuarto del siglo $\mathrm{XX}$, muchos autores han defendido que este análisis nos lo hemos de plantear desde la perspectiva del trípode socializador básico de las sociedades contemporáneas: familia-escuela-televisión. Consideramos que más que hablar tan sólo de la televisión, hoy en día catalogada como "vieja/antigua tecnología", es más adecuado referirnos a "las nuevas pantallas" (ordenador, videojuegos, Internet, teléfono móvil) ya que estas tecnologías han adquirido gran protagonismo y relevancia en buena parte gracias a los niños/as y los/las adolescentes (Malo, 2009).

Podemos apuntar que en la adolescencia, al trípode socializador le podríamos añadir un cuarto agente socializador: el grupo de iguales. En este mismo sentido, diferentes autores europeos señalan la posibilidad que hayamos infra-valorado las relaciones entre iguales (Suoninen, 2001). Parece ser que la televisión y las nuevas tecnologías juegan un papel importante, e incluso creciente, influyendo sobre los niños/as y adolescentes europeos de entre 6 y 16 años no tanto directamente como a través de las relaciones que mantienen con los compañeros de su misma edad (Suess et al., 1998).

Las investigaciones sobre los procesos de socialización paterno-filiales centradas en la perspectiva relacional, ponen de manifiesto como las relaciones entre los progenitores y los hijos/as son recíprocas y más dinámicas de lo que se pensaba desde los modelos de socialización más tradicionales (Lollis y Kuczynski, 1997; Maccoby y Martin, 1983). Un elemento central de esta perspectiva tiene que ver con el hecho que los niños/as y los/las adolescentes se socializan participando en las relaciones interpersonales más cercanas, más íntimas (Kuczynski, 2003; Reiss, Collins y Berscheild, 2000).

Aunque, como señalan Cantero y Gordillo (1999), los progenitores y los hijos/as se comunican de forma más abierta en la actualidad, conversan más y discuten las decisiones que afecten a los hijos/as, no parece ser que hablar sobre las TICs esté entre sus temas preferidos. Según los autores, los temas que más se abordan son los relativos a la vida familiar, especialmente aquellos que tienen que ver con la organización y las normas. Así mismo lo constata Malo (2009), señalando que los progenitores priorizan hablar con sus hijos/as adolescentes temas relativos a sus hábitos de vida cotidiana (los estudios, las normas familiares, entre otros) más que hablar sobre tecnologías.

Los estudios realizados por Casas y sus colaboradores desde hace más de diez años, señalan que existe una tendencia generalizada de los adultos a no aceptar que, a veces, son menos competentes que los niños/as o los/las adolescentes en relación a los medios audiovisuales (Casas, 2001). En este sentido, antes de aceptar este hecho, muchos adultos prefieren (a) evitar la comunicación sobre el tema o (b) desvalorizar las competencias infantiles. Muchos adultos consideran que ellos tienen mucho que ensenar y poco que aprender. No es posible que los aún no competentes puedan enseñar cosas a los ya sí competentes.

Estos mismos resultados se constatan en investigaciones realizadas con una muestra de adolescentes 
y progenitores catalanes (Casas, Alsinet et al., 2000) y con datos comparativos de cinco países (Casas, Mjaavatn et al., 2007): los chicos y las chicas se sienten más satisfechos/as cuando mantienen conversaciones sobre medios audiovisuales con los amigos/as y menos satisfechos/as cuando lo hacen con los adultos en general, tanto con los progenitores, como con los profesores/as.

La presente investigación tiene por objetivo explorar a través de la frecuencia y la satisfacción con las conversaciones sobre TICs qué cambios se han producido en un período de cinco años, en la relación de los chicos y chicas de 12 a 16 años y sus progenitores.

\section{Método}

\section{Muestra $2003^{1}$}

Para obtener la muestra de adolescentes se realizó un muestreo por conglomerados a partir de la selección de institutos de secundaria de entornos sociodemográficos distintos de las cuatro provincias de Cataluña.

La muestra resultante está formada por 1.104 adolescentes de entre 12 y 16 años, todos ellos estudiantes de ESO en centros de titularidad pública. El $44,6 \%$ de los adolescentes participantes son chicos y el $55,4 \%$ restante chicas, siendo la media de edad de 13,6 años $(\mathrm{dt}=1,268)$.

La muestra de progenitores se obtuvo mediante los adolescentes participantes, a quienes se les facilitó un cuestionario para que lo entregasen a sus padres y lo devolvieran al instituto una vez cumplimentado.

\section{Muestra 2008}

Esta otra muestra se seleccionó mediante un muestreo estratificado por centros de titularidad pública-concertada y entorno urbano-semiurbanorural, de las provincias de Girona y Lleida.

\footnotetext{
${ }^{1}$ Debido a que el artículo tiene como objetivo relacionar respuestas entre padres e hijos/as no se han tenido en cuenta los casos de los que no se disponía de la información de los padres.
}

La muestra obtenida es de 1.370 adolescentes, todos ellos estudiantes de ESO en centros públicos y privados. Los chicos representan el $45,8 \%$ de la muestra y las chicas el $54,2 \%$ restante, con una media de edad de 14,1 años ( $\mathrm{dt}=1,277)$.

Para obtener la muestra de los progenitores se utilizó el mismo método de recogida que en la muestra del 2003.

\section{Procedimiento}

El procedimiento seguido en las dos recogidas de datos fue el mismo. En un primer momento se contactó con los centros educativos y se informó de la investigación al director/a y al presidente/a de la asociación de padres y madres. Una vez obtenido el permiso para la administración de los cuestionarios, se concertó con los jefes de estudios y los docentes los días y las horas más adecuadas.

El cuestionario se administró de forma grupal en el aula habitual de los chicos y chicas, con la presencia del profesor/a responsable de la clase en aquel momento y la de como mínimo un investigador/a para informar a los/las adolescentes de la investigación, dejando claro que sus respuestas serían tratadas confidencialmente, y para resolver las dudas que pudieran surgir. Cada sesión tenía previsto durar en torno a una hora.

Al final de la sesión entregamos a cada alumno/a una carta y un cuestionario para sus progenitores en un sobre, para que lo entregaran en mano. Les solicitamos que lo retornaran a los profesores/as en el término de una semana, también en el sobre cerrado. El cuestionario de progenitores tenía un código, para poder emparejarlo con el de cada hijo/a.

\section{Instrumentos}

En la muestra del 2003 el instrumento utilizado para la recogida de datos fue un cuestionario diseñado para el estudio del uso de medios audiovisuales y la comunicación sobre ellos de los adolescentes y sus progenitores, y su relación con el bienestar psicológico. 
Para la muestra del 2008 también se utilizó un cuestionario, con escalas psicométricas de bienestar psicológico y constructos psicosociales relacionados con este concepto, además de algunos ítems relacionados con las TICs y el tiempo libre.

Aunque los dos cuestionarios son distintos, comparten las mismas variables relacionadas con las tecnologías de la información y comunicación, lo que permite su comparación.

\section{Definición de las variables}

\section{Adolescentes}

a) Disponibilidad de las diferentes tecnologías estudiadas por parte del/de la adolescente.

Se pidió a los/las adolescentes que valorasen a partir de una escala dicotómica Sí o No, si disponían de una serie de medios audiovisuales (televisor, ordenador, Internet, videoconsola, videojuegos para el ordenador y teléfono móvil), preguntándoles si eran suyos, si estaban en su casa pero no eran suyos y los utilizaba o si utilizaba otros fuera de su casa.

b) Frecuencia con la que el/la adolescente dice hablar con cada uno de sus progenitores, sus hermanos/as, amigos/as y profesores/as de su experiencia con las tecnologías.

Para explorar la frecuencia con la que los/las adolescentes hablan con distintas personas (progenitores, hermanos/as, amigos/as y profesores/as) sobre el conjunto de medios audiovisuales estudiados (televisión, ordenador, Internet, videojuegos y móvil), se utilizó una escala Likert de 5 puntos, donde Nunca es 0 y Muy a menudo es 5.

c) Satisfacción que el/la adolescente informa tener cuando mantiene conversaciones con el padre, la madre, los amigos/as y los profesores/as sobre las diferentes tecnologías estudiadas (TV, ordenador, Internet y juegos).

La satisfacción que el adolescente informa tener cuando habla sobre las diferentes medios audiovi- suales estudiados (televisión, ordenador, Internet y videojuegos) con el padre, la madre, los amigos/as y los profesores/as, se exploró mediante una escala Likert de 5 puntos (1 No me gusta nada y $5 \mathrm{Me}$ gusta muchísimo), además de plantearse la opción Nunca hablo de esto.

\section{Progenitores}

a) Atribución del progenitor a la satisfacción que su hijola tiene cuando mantiene conversaciones con el padre, la madre, los amigos/as y los profesores/as sobre las diferentes tecnologías estudiadas (TV, ordenador, Internet y juegos).

Se exploró la atribución del progenitor a la satisfacción de su hijo/a respecto a la comunicación con distintas personas sobre las cosas que hacen o miran con cada uno de los medios audiovisuales mediante una escala tipo Likert de 5 puntos, donde 1 equivale a No le gusta nada y 5 a Le gusta muchísimo. A los progenitores también se consideraba la opción de respuestas: Nunca habla de esto.

\section{Resultados}

Con el objetivo de explorar las diferencias entre 2003 y 2008 en las respuestas dadas a las variables estudiadas, se han realizado pruebas chi-cuadrado para aquellas variables dicotómicas y t de Student para las escalas. Se han analizado por un lado las respuestas obtenidas de los/las adolescentes y por el otro las obtenidas de sus progenitores.

\section{Respuestas de los/las adolescentes}

Disponibilidad de las diferentes tecnologías estudiadas por parte del/de la adolescente

Se han obtenido diferencias significativas en relación a todas las tecnologías estudiadas (en este caso $\mathrm{TV}$, ordenador, Internet, videoconsola, juegos de ordenador y móvil) excepto en los juegos de ordenador $\left(\chi_{1}^{2}=2,75 ; p=0,054\right)$. Así, mientras la disponibili- 
dad de un televisor propio $\left(\chi_{1}^{2}=14,718 ; \mathrm{p}<0,0005\right)$ por parte de los adolescentes era mayor en el año 2003, en el año 2008 un mayor número de adolescentes dispone de ordenador $\left(\chi_{1}^{2}=4,989 ; \mathrm{p}=0,014\right)$, conexión a Internet $\left(\chi_{1}^{2}=79,504 ; \mathrm{p}<0,0005\right)$, videoconsola $\left(\chi_{1}^{2}=10,807 ; \mathrm{p}=0,001\right)$ y móvil $\left(\chi_{1}^{2}=8,078\right.$; $\mathrm{p}=0,003)$ que consideran propios.

Frecuencia con la que el/la adolescente dice hablar con cada uno de sus progenitores, sus hermanos/as, amigos/as y profesores/as de su experiencia con las tecnologías.

En relación a la frecuencia con la que los/las adolescentes mantienen conversaciones con las personas de su entorno relativas a las TIC, observamos que no existe diferencia entre el 2003 y el 2008 en la frecuencia de estas conversaciones cuando el interlocutor es un profesor/a $\left(\mathrm{t}_{1355}=1,949 ; \mathrm{p}=0,051\right)$, pero sí las hay cuando lo es el padre $\left(\mathrm{t}_{1410}=7,027\right.$; $\mathrm{p}<0,0005)$, la madre $\left(\mathrm{t}_{1749}=7,16 ; \mathrm{p}<0,0005\right)$, los hermanos/as $\left(\mathrm{t}_{1287}=4,878 ; \mathrm{p}<0,0005\right)$ y los amigos/as, tanto los de igual edad $\left(\mathrm{t}_{1493}=2,589 ; \mathrm{p}=0,01\right)$, como los mayores $\left(\mathrm{t}_{1151}=2,977 ; \mathrm{p}=0,003\right)$. En todos los casos, la mayor frecuencia se da en el 2008.

Satisfacción del/de la adolescente cuando mantiene conversaciones con el padre, la madre, los amigos/as y los profesores/as sobre las diferentes tecnologías estudiadas (TV, ordenador, Internet y videojuegos).

Los/las adolescentes de la muestra de 2003 se muestran significativamente más satisfechos que los de la muestra de 2008 con las conversaciones man- tenidas con el padre, la madre y los/las profesores/as, relativas a todas las tecnologías estudiadas, exceptuando las conversaciones sobre TV con la madre en las que no se observa diferente nivel de satisfacción entre las muestras.

Cuando se analiza la satisfacción de estas conversaciones con los/las amigos/as, observamos que en las relativas a los videojuegos, la satisfacción experimentada no es significativamente diferente $\left(\mathrm{t}_{1622}=1,425 ; \mathrm{p}=0,154\right)$ entre el 2003 y el 2008 , mientras sí lo es en las conversaciones relativas a la TV $\left(\mathrm{t}_{1369}=2,091 ; \mathrm{p}=0,037\right)$, el ordenador $\left(\mathrm{t}_{1524}=2,486\right.$; $\mathrm{p}=0,013)$ e Internet $\left(\mathrm{t}_{1561}=2,624 ; \mathrm{p}=0,009\right)$. Dándose en el primer caso mayor satisfacción en el año 2003 y en los otros dos mayor satisfacción en 2008.

Relación entre la frecuencia en las conversaciones y satisfacción que producen.

Al correlacionar la frecuencia y la satisfacción producida por las conversaciones relativas a las diferentes TICs estudiadas, observamos que en el 2008 la satisfacción con las conversaciones sobre TV y sobre ordenadores correlaciona positivamente con la frecuencia de éstas, ya sean con el padre o con la madre. En el 2003 la frecuencia y la satisfacción de las conversaciones con el padre correlacionan de forma negativa si se dan en torno a Internet, y con la madre las relacionadas con la TV correlacionan de forma positiva y con los videojuegos de forma negativa.

Cuando preguntamos por las conversaciones mantenidas con algún profesor o profesora acerca de estas TIC, no se da relación alguna entre la frecuencia y la satisfacción producida, en ninguna de las 2 muestras.

Tabla 1. Satisfacción de los/las adolescentes al mantener conversaciones con los adultos en relación a diferentes tecnologías

\begin{tabular}{|c|c|c|c|}
\hline & Padre & Madre & Profesor/a \\
\hline TV & $\begin{array}{l}\mathrm{t}_{1487}=2,8 \\
\mathrm{p}=0,005\end{array}$ & $\begin{array}{c}\mathrm{t}_{1423}=0,964 \\
\mathrm{p}=0,335\end{array}$ & $\begin{array}{c}\mathrm{t}_{1370}=6,828 \\
\mathrm{p}<0,0005\end{array}$ \\
\hline Ordenador & $\begin{array}{c}\mathrm{t}_{1511}=4,633 \\
\mathrm{p}<0,0005\end{array}$ & $\begin{array}{c}\mathrm{t}_{1485}=2,712 \\
\mathrm{p}=0,007\end{array}$ & $\begin{array}{c}\mathrm{t}_{1372}=6,861 \\
\mathrm{p}<0,0005\end{array}$ \\
\hline Internet & $\begin{array}{c}\mathrm{t}_{1523}=3,531 \\
\mathrm{p}<0,0005\end{array}$ & $\begin{array}{c}\mathrm{t}_{1501}=3,164 \\
\mathrm{p}=0,002\end{array}$ & $\begin{array}{c}\mathrm{t}_{1408}=7,081 \\
\mathrm{p}<0,0005\end{array}$ \\
\hline Videojuegos & $\begin{array}{c}\mathrm{t}_{1430}=4,023 \\
\mathrm{p}<0,0005\end{array}$ & $\begin{array}{c}\mathrm{t}_{1395}=3,338 \\
\mathrm{p}=0,001\end{array}$ & $\begin{array}{c}\mathrm{t}_{1361}=6,409 \\
\mathrm{p}<0,0005\end{array}$ \\
\hline
\end{tabular}


Tabla 2. Correlación entre la frecuencia de las conversaciones que se mantienen sobre tecnologías con el padre y la madre, y la satisfacción que producen las mismas

\begin{tabular}{|c|c|c|c|c|}
\hline & \multicolumn{2}{|c|}{2003} & \multicolumn{2}{|c|}{2008} \\
\hline & Padre & Madre & Padre & Madre \\
\hline TV & $\begin{array}{l}r=0,031 \\
p=0,333\end{array}$ & $\begin{array}{l}\mathrm{r}=0,083 \\
\mathrm{p}=0,009\end{array}$ & $\begin{array}{c}r=0,203 \\
\mathrm{p}<0,0005\end{array}$ & $\begin{array}{c}\mathrm{r}=0,148 \\
\mathrm{p}<0,0005\end{array}$ \\
\hline Ordenador & $\begin{array}{l}r=-0,6 \\
p=0,06\end{array}$ & $\begin{array}{c}\mathrm{r}=-0,034 \\
\mathrm{p}=0,29\end{array}$ & $\begin{array}{l}r=0,113 \\
p=0,005\end{array}$ & $\begin{array}{l}r=0,112 \\
p=0,005\end{array}$ \\
\hline Internet & $\begin{array}{l}r=-0,086 \\
p=0,007\end{array}$ & $\begin{array}{l}r=-0,043 \\
p=0,181\end{array}$ & $\begin{array}{l}r=0,073 \\
p=0,07\end{array}$ & $\begin{array}{l}r=0,073 \\
p=0,068\end{array}$ \\
\hline Videojuegos & $\begin{array}{l}r=-0,05 \\
p=0,124\end{array}$ & $\begin{array}{l}r=-0,078 \\
p=0,016\end{array}$ & $\begin{array}{l}r=-0,027 \\
p=0,504\end{array}$ & $\begin{array}{l}r=-0,035 \\
p=0,389\end{array}$ \\
\hline
\end{tabular}

Tabla 3. Correlación entre la frecuencia de las conversaciones que se mantienen sobre tecnologías con algún profesor/a y la satisfacción que producen las mismas

\begin{tabular}{lcc}
\hline & 2003 & 2008 \\
\hline TV & $\mathrm{r}=-0,053$ & $\mathrm{r}=-0,057$ \\
& $\mathrm{p}=0,1$ & $\mathrm{p}=0,158$ \\
\hline Ordenador & $\mathrm{r}=-0,06$ & $\mathrm{r}=-0,044$ \\
& $\mathrm{p}=0,064$ & $\mathrm{p}=0,275$ \\
\hline Internet & $\mathrm{r}=-0,061$ & $\mathrm{r}=-0,026$ \\
& $\mathrm{p}=0,059$ & $\mathrm{p}=0,513$ \\
\hline Videojuegos & $\mathrm{r}=-0,016$ & $\mathrm{r}=-0,077$ \\
& $\mathrm{p}=0,621$ & $\mathrm{p}=0,056$
\end{tabular}

La frecuencia y la satisfacción de las conversaciones con los amigos/as correlacionan positivamente si son acerca de TV, ordenador o Internet, tanto con amigos/as de la misma edad como con los mayores en 2008 y sólo con los de la misma edad en 2003.

\section{Respuestas de los progenitores}

Atribución del progenitor a la satisfacción de su hijola cuando mantiene conversaciones con el padre, la madre, los amigos/as y los profesores/as

Tabla 4. Correlación entre la frecuencia de las conversaciones que se mantienen sobre tecnologías con los/las amigos/as y la satisfacción que producen las mismas

\begin{tabular}{|c|c|c|c|c|}
\hline & & & & \\
\hline & Amigos/as de igual edad & Amigos/as mayores & Amigos/as de igual edad & Amigos/as mayores \\
\hline TV & $\mathrm{r}=0,147$ & $r=0,07$ & $\mathrm{r}=0,188$ & $r=0,089$ \\
\hline & $\mathrm{p}<0,0005$ & $\mathrm{p}=0,138$ & $\mathrm{p}<0,0005$ & $\mathrm{p}=0,026$ \\
\hline Ordenador & $\mathrm{r}=0,105$ & $r=0,04$ & $\mathrm{r}=0,137$ & $\mathrm{r}=0,101$ \\
\hline & $\mathrm{p}=0,001$ & $\mathrm{p}=0,398$ & $\mathrm{p}=0,001$ & $\mathrm{p}=0,011$ \\
\hline Internet & $\mathrm{r}=0,182$ & $\mathrm{r}=0,077$ & $\mathrm{r}=0,184$ & $\mathrm{r}=0,104$ \\
\hline & $\mathrm{p}<0,0005$ & $\mathrm{p}=0,104$ & $\mathrm{p}<0,0005$ & $\mathrm{p}=0,01$ \\
\hline Videojuegos & $\mathrm{r}=0,031$ & $\mathrm{r}=-0,057$ & $\mathrm{r}=-0,036$ & $\mathrm{r}=-0,021$ \\
\hline & $\mathrm{p}=0,335$ & $\mathrm{p}=0,228$ & $\mathrm{p}=372$ & $\mathrm{p}=0,594$ \\
\hline
\end{tabular}


sobre las diferentes tecnologías estudiadas (TV, ordenador, Internet y videojuegos).

En 2003, los progenitores atribuían a su hijo/a adolescente más satisfacción con todas las conversaciones mantenidas, independientemente de la persona con quien se mantienen y de la tecnología en cuestión. La única excepción son las relativas a la TV con la madre, en las que no se observan diferencias significativas entre muestras.
En las dos muestras estudiadas, adolescentes y progenitores coinciden al puntuar la satisfacción que experimenta el/la adolescente cuando mantiene conversaciones con el padre relativas a todas las tecnologías estudiadas, excepto al ordenador, para el que la atribución de satisfacción que realizan los progenitores es significativamente más elevada que la propia satisfacción de la que informa su hijo o hija. Observamos no obstante, que existe una correlación positiva entre la satisfacción manifestada por

Tabla 5. Atribución del progenitor a la satisfacción de su hijo/a cuando mantiene conversaciones con diferentes personas sobre las diferente tecnologías estudiadas

\begin{tabular}{|c|c|c|c|c|}
\hline & Padre & Madre & Profesor/a & Amigos/as \\
\hline TV & $\begin{array}{c}\mathrm{t}_{594}=2,936 \\
\mathrm{p}=0,003\end{array}$ & $\begin{array}{c}\mathrm{t}_{683}=0,177 \\
\mathrm{p}=0,86\end{array}$ & $\begin{array}{c}\mathrm{t}_{577}=13,171 \\
\mathrm{p}<0,0005\end{array}$ & $\begin{array}{l}\mathrm{t}_{580}=5,737 \\
\mathrm{p}<0,0005\end{array}$ \\
\hline Ordenador & $\begin{array}{c}\mathrm{t}_{599}=2,862 \\
\mathrm{p}=0,004\end{array}$ & $\begin{array}{l}\mathrm{t}_{652}=3,48 \\
\mathrm{p}=0,001\end{array}$ & $\begin{array}{c}\mathrm{t}_{556}=12,339 \\
\mathrm{p}<0,0005\end{array}$ & $\begin{array}{c}\mathrm{t}_{539}=2,094 \\
\mathrm{p}=0,037\end{array}$ \\
\hline Internet & $\begin{array}{c}t_{567}=2,594 \\
p=0,01\end{array}$ & $\begin{array}{c}\mathrm{t}_{550}=12,528 \\
\mathrm{p}<0,0005\end{array}$ & $\begin{array}{l}\mathrm{t}_{604}=3,541 \\
\mathrm{p}<0,0005\end{array}$ & $\begin{array}{c}\mathrm{t}_{523}=2,484 \\
\mathrm{p}=0,013\end{array}$ \\
\hline Videojuegos & $\begin{array}{c}\mathrm{t}_{\mathrm{t} 26}=3,162 \\
\mathrm{p}=0,002\end{array}$ & $\begin{array}{l}\mathrm{t}_{645}=3,89 \\
\mathrm{p}<0,0005\end{array}$ & $\begin{array}{c}t_{600}=12,353 \\
p<0,0005\end{array}$ & $\begin{array}{c}\mathrm{t}_{604}=2,6866 \\
\mathrm{p}=0,007\end{array}$ \\
\hline
\end{tabular}

\section{Respuestas familiares: coincidencia $y$ discrepancia entre las percepciones de adolescentes y progenitores}

Satisfacción del/de la adolescente y atribución de satisfacción del progenitor a esta satisfacción con las conversaciones con el padre, la madre, los amigos/as y los profesores/as sobre las diferentes tecnologías estudiadas. los/las adolescentes y la atribución que sus progenitores hacen, sea cual sea la tecnología a la que se refieren.

Cuando la interlocutora en esas conversaciones es la madre, no existen diferencias significativas en las puntuaciones medias de las respuestas sobre satisfacción dadas por adolescentes y sus progenitores, para ninguna de las tecnologías, ni en ninguna de las dos muestras. En este caso, y a diferencia de lo que

Tabla 6. Comparación entre la satisfacción del/de la adolescente con las conversaciones con su padre y la atribución de satisfacción de los progenitores sobre las diferentes tecnologías estudiadas

\begin{tabular}{|c|c|c|c|c|}
\hline \multicolumn{3}{|c|}{2003} & \multicolumn{2}{|c|}{2008} \\
\hline $\begin{array}{l}\text { TV } \\
p=0,415\end{array}$ & $\begin{array}{c}\mathrm{t}_{394}=0,816 \\
\mathrm{p}=0,006\end{array}$ & $\begin{array}{l}r=0,137 \\
p=0,838\end{array}$ & $\begin{array}{c}\mathrm{t}_{574}=0,205 \\
\mathrm{p}=0,01\end{array}$ & $\mathrm{r}=0,108$ \\
\hline Ordenador & $\begin{array}{c}\mathrm{t}_{389}=2,579 \\
\mathrm{p}=0,01\end{array}$ & $\begin{array}{l}r=0,157 \\
p=0,002\end{array}$ & $\begin{array}{c}\mathrm{t}_{570}=2,928 \\
\mathrm{p}=0,004\end{array}$ & $\begin{array}{c}r=0,151 \\
\mathrm{p}<0,0005\end{array}$ \\
\hline Internet & $\begin{array}{c}\mathrm{t}_{375}=0,877 \\
\mathrm{p}=0,381\end{array}$ & $\begin{array}{l}r=0,122 \\
p=0,018\end{array}$ & $\begin{array}{c}\mathrm{t}_{569}=0,854 \\
\mathrm{p}=0,394\end{array}$ & $\begin{array}{c}r=0,151 \\
\mathrm{p}<0,0005\end{array}$ \\
\hline Videojuegos & $\begin{array}{c}\mathrm{t}_{379}=1,632 \\
\mathrm{p}=0,104\end{array}$ & $\begin{array}{l}\mathrm{r}=0,12 \\
\mathrm{p}=0,02\end{array}$ & $\begin{array}{c}\mathrm{t}_{566}=1,549 \\
\mathrm{p}=0,122\end{array}$ & $\begin{array}{l}\mathrm{r}=0,115 \\
\mathrm{p}=0,006\end{array}$ \\
\hline
\end{tabular}


pasaba en la satisfacción de las conversaciones con el padre, no se observa sistemáticamente una tendencia similar de respuesta entre unos y otros. Las correlaciones positivas se dan en las conversaciones sobre la televisión y los juegos de ordenador, para eran los progenitores los que consideraban que la satisfacción de su hijo/a con las conversaciones sobre ordenador e Internet con amigos/as era significativamente más elevada que la propia satisfacción que el/la adolescente expresaba, y únicamente

Tabla 7. Comparación entre la satisfacción del/de la adolescente con las conversaciones con su madre y la atribución de satisfacción de los progenitores sobre las diferentes tecnologías estudiadas

\begin{tabular}{|c|c|c|c|c|}
\hline \multirow[b]{2}{*}{ TV } & \multicolumn{2}{|c|}{2003} & \multicolumn{2}{|c|}{2008} \\
\hline & $\begin{array}{c}\mathrm{t}_{411}=0,897 \\
\mathrm{p}=0,37\end{array}$ & $\begin{array}{l}r=0,107 \\
p=0,03\end{array}$ & $\begin{array}{c}\mathrm{t}_{584}=0,947 \\
\mathrm{p}=0,344\end{array}$ & $\begin{array}{c}\mathrm{r}=0,167 \\
\mathrm{p}<0,0005\end{array}$ \\
\hline Ordenador & $\begin{aligned} \mathrm{t}_{399} & =1,840 \\
\mathrm{p} & =0,066\end{aligned}$ & $\begin{array}{l}\mathrm{r}=0,085 \\
\mathrm{p}=0,09\end{array}$ & $\begin{aligned} t_{584} & =1,699 \\
p & =0,09\end{aligned}$ & $\begin{array}{l}\mathrm{r}=0,081 \\
\mathrm{p}=0,051\end{array}$ \\
\hline Internet & $\begin{array}{c}\mathrm{t}_{386}=0,312 \\
\mathrm{p}=0,755\end{array}$ & $\begin{array}{l}\mathrm{r}=0,142 \\
\mathrm{p}=0,005\end{array}$ & $\begin{array}{c}\mathrm{t}_{582}=0,126 \\
\mathrm{p}=0,9\end{array}$ & $\begin{array}{c}\mathrm{r}=0,08 \\
\mathrm{p}=0,053\end{array}$ \\
\hline Videojuegos & $\begin{array}{c}t_{378}=1,585 \\
p=0,114\end{array}$ & $\begin{array}{l}\mathrm{r}=0,156 \\
\mathrm{p}=0,002\end{array}$ & $\begin{array}{c}t_{572}=1,651 \\
p=0,099\end{array}$ & $\begin{array}{l}r=0,087 \\
p=0,037\end{array}$ \\
\hline
\end{tabular}

ambas muestras y sobre Internet en la muestra de 2003.

$\mathrm{Al}$ analizar la satisfacción experimentada por y atribuida a los/las adolescentes cuando conversan sobre las TIC con sus amigos/as, observamos algunas diferencias entre muestras. Así, en 2008 los y las adolescentes valoran las conversaciones sobre la TV con sus amigos/as como significativamente más satisfactorias de lo que sus progenitores les atribuían, y encontramos que las respuestas de unos y otros correlacionan positivamente, mostrando una pauta de respuesta con la misma tendencia entre progenitores e hijos/as. En 2003, en cambio, se observa correlación positiva cuando las conversaciones se refieren a Internet.

Cuando las conversaciones se mantienen con algún profesor o profesora, observamos muchas más diferencias en las respuestas de adolescentes y progenitores. Así, la satisfacción atribuida por los padres y madres a estas conversaciones era significativamente más elevada que la propia satisfacción informada por el/la adolescentes, para todas las tecnologías estudiadas en 2003 y para ordenador e Internet en 2008. Y únicamente hallamos correlación positiva en las respuestas para las relacionadas con la TV.

Tabla 8. Comparación entre la satisfacción del/de la adolescente con las conversaciones con sus amigos/as y la atribución de satisfacción de los progenitores sobre las diferentes tecnologías estudiadas

\begin{tabular}{|c|c|c|c|c|}
\hline \multicolumn{3}{|c|}{2003} & \multicolumn{2}{|c|}{2008} \\
\hline TV & $\begin{array}{c}\mathrm{t}_{405}=1,485 \\
\mathrm{p}=0,138\end{array}$ & $\begin{array}{l}r=0,033 \\
p=0,507\end{array}$ & $\begin{array}{l}\mathrm{t}_{572}=3,625 \\
\mathrm{p}<0,0005\end{array}$ & $\begin{array}{c}r=0,15 \\
p<0,0005\end{array}$ \\
\hline Ordenador & $\begin{array}{c}\mathrm{t}_{400}=3,396 \\
\mathrm{p}=0,001\end{array}$ & $\begin{array}{l}\mathrm{r}=0,033 \\
\mathrm{p}=0,513\end{array}$ & $\begin{array}{c}\mathrm{t}_{573}=0,318 \\
\mathrm{p}=0,751\end{array}$ & $\begin{array}{c}\mathrm{r}=0,287 \\
\mathrm{p}<0,0005\end{array}$ \\
\hline Internet & $\begin{array}{c}\mathrm{t}_{387}=2,305 \\
\mathrm{p}=0,022\end{array}$ & $\begin{array}{l}r=0,102 \\
p=0,046\end{array}$ & $\begin{array}{c}\mathrm{t}_{574}=0,557 \\
\mathrm{p}=0,578\end{array}$ & $\begin{array}{c}r=0,189 \\
\mathrm{p}<0,0005\end{array}$ \\
\hline Videojuegos & $\begin{array}{c}\mathrm{t}_{392}=1,271 \\
\mathrm{p}=0,204\end{array}$ & $\begin{array}{l}r=0,087 \\
p=0,083\end{array}$ & $\begin{array}{c}\mathrm{t}_{567}=0,041 \\
\mathrm{p}=0,967\end{array}$ & $\begin{array}{c}r=0,219 \\
p<0,0005\end{array}$ \\
\hline
\end{tabular}


Tabla 9. Comparación entre la satisfacción del/de la adolescente con las conversaciones con algún profesor/a y la atribución de satisfacción de los progenitores sobre las diferentes tecnologías estudiadas

\begin{tabular}{|c|c|c|c|c|}
\hline \multirow[b]{2}{*}{ TV } & \multicolumn{2}{|c|}{2003} & \multicolumn{2}{|c|}{2008} \\
\hline & $\begin{array}{l}\mathrm{t}_{387}=5,018 \\
\mathrm{p}<0,0005\end{array}$ & $\begin{array}{l}r=0,108 \\
p=0,033\end{array}$ & $\begin{array}{c}\mathrm{t}_{545}=1,805 \\
\mathrm{p}=0,072\end{array}$ & $\begin{array}{l}r=0,019 \\
p=0,66\end{array}$ \\
\hline Ordenador & $\begin{array}{l}\mathrm{t}_{377}=5,964 \\
\mathrm{p}<0,0005\end{array}$ & $\begin{array}{l}r=0,049 \\
p=0,337\end{array}$ & $\begin{array}{l}\mathrm{t}_{544}=3,799 \\
\mathrm{p}<0,0005\end{array}$ & $\begin{array}{l}r=0,057 \\
p=0,185\end{array}$ \\
\hline Internet & $\begin{array}{l}\mathrm{t}_{377}=5,679 \\
\mathrm{p}<0,0005\end{array}$ & $\begin{array}{l}\mathrm{r}=0,085 \\
\mathrm{p}=0,101\end{array}$ & $\begin{array}{c}\mathrm{t}_{549}=4,03 \\
\mathrm{p}<0,0005\end{array}$ & $\begin{array}{l}r=0,077 \\
p=0,072\end{array}$ \\
\hline Videojuegos & $\begin{array}{l}\mathrm{t}_{375}=3,717 \\
\mathrm{p}<0,0005\end{array}$ & $\begin{array}{c}\mathrm{r}=0,1 \\
\mathrm{p}=0,052\end{array}$ & $\begin{array}{c}\mathrm{t}_{540}=0,189 \\
\mathrm{p}=0,843\end{array}$ & $\begin{array}{l}r=0,028 \\
p=0,519\end{array}$ \\
\hline
\end{tabular}

\section{Discusión}

Los resultados obtenidos respecto a la frecuencia con la que los/las adolescentes y sus progenitores hablan sobre las cosas que hacen y miran utilizando las TIC se mantienen en la misma línea de los hallados anteriormente. Además, observamos que, en general, resultan conversaciones poco frecuentes y valoradas como poco satisfactorias. No obstante, se observa también que en el transcurso de los cinco años que separan las dos muestras estudiadas ha aumentado la frecuencia de conversaciones con los progenitores y disminuido aún más la satisfacción. Parece que los adultos han asumido la necesidad de implicarse y buscan conversaciones relativas a temas y actividades que motivan e interesan a sus hijos/as como son las TICs, persiguiendo un acercamiento intergeneracional.

Sin embargo, las conversaciones mantenidas son vividas como menos satisfactorias que 5 años atrás. La disminución de la satisfacción no es únicamente manifestada por los/las adolescentes, sino que sus propios progenitores lo perciben, atribuyendo también menos satisfacción en las conversaciones.

En la muestra de 2003, las correlaciones negativas entre la frecuencia y la satisfacción de las conversaciones con el padre y la madre (sobre Internet con el padre y sobre videojuegos con la madre) parecen mostrar que el aumento en la frecuencia, hace aumentar también la percepción de los/las adolescentes de que su padre o madre no domina el tema, o de que conversa con él/ella desde el desconocimiento, por lo que aumenta la insatisfacción. Como señala Malo (2009), una de las razones considerada un obstáculo para la comunicación acerca del uso de las tecnologías es el desconocimiento de los progenitores en relación a algunas TICs, aludido tanto por los/las adolescentes, como por los propios progenitores.

La poca correlación observada en las dos muestras entre la satisfacción informada por los/las adolescentes y la atribuida por sus progenitores cuando mantienen conversaciones con los adultos (padre, madre o profesores/as), apuntaría a que los progenitores no tienen una percepción congruente a la satisfacción real que su hijo/a manifiesta. Por otro lado, cuando exploramos las conversaciones mantenidas con los amigos/as, las correlaciones elevadas entre la satisfacción de los/las adolescentes y la atribución de sus progenitores, parecen mostrar una percepción más congruente.

La baja satisfacción manifestada al mantener conversaciones con los adultos en torno a las TICs, sumada a los sesgos perceptivos de los progenitores, sugieren la necesidad de un acercamiento de intereses intergeneracionales que consideramos debería producirse por ambas partes: los/las adolescentes aportando su experiencia en el uso de las tecnologías y los adultos como referentes en su proceso de socialización. Parece ser que los resultados corroboran esta idea: se podría decir que los progenitores están cada vez más dispuestos a asumir su papel, mientras que los/las adolescentes responden al esfuerzo que realizan sus progenitores para interesarse por aquellas cosas que los motivan.

Se observan correlaciones positivas entre frecuencia y satisfacción de las conversaciones con padre y madre en el 2008, apuntando a un cambio de 
actitud de algunos progenitores en relación a la aportación de las TICs al desarrollo psicosocial de su hijo/a. Éstos, en la línea de las ideas aportadas por Tapscott (1998), considerarían que las tecnologías son positivas para el desarrollo de niños/as y adolescentes o se sitúan en la línea de lo que Bressand y Distler (1995) denominan "integrados" a los avances tecnológicos. En todo caso, este cambio de actitud permite un aumento en la frecuencia de las conversaciones entre padres e hijos que conlleva una disminución de las diferencias intergrupales sobre las TICs (Casas, 2006).

También se observa un cambio en la relación con los amigos/as. Así, mientras en el 2003 los resulta- dos muestran que la relación con los amigos de más edad era más próxima a la relación con los adultos, en 2008 esta relación es más próxima a la relación con los amigos/as de igual edad. Esto indicaría que el grupo de amigos/as es considerado homogéneo independientemente de la edad pudiendo ser el dominio de las TIC un criterio de satisfacción con sus conversaciones. Se evidencia una fuerte identificación social con el grupo de amigos/as, confrontada con el grupo de adultos, cosa que también se relacionaría con uno de los principios de la GS Theory formulados por Harris (1995), según el cual los/las adolescentes fuera del entorno familiar se identifican con el grupo de iguales.

\section{Extended Summary}

\section{Introduction}

New social dynamics and quick technological advances have produced changes in the interactions between adults and adolescents, as could be the infantilization of adolescence that lead them to difficulties to access to adult world, or the changes in the way new social and family values are understood and interpreted. ICTs seem to increase categorical differentiation between adolescents and adults, also producing the so-called generational gap: adolescents, and even children, are becoming actual experts using electronic media and the socialization in this field is not produced with adults but with peers outside the familiar environment. Children and adolescents learn to use media with peers who take the place of parents, probably because of the interest of adults in maintaining children and adolescents in the separated category of "non-adults" instead of deepen in socialization processes and construction of new social consensus with new generations.

The importance of "new-screens" as a socializing agent have been defended in the last quarter of $20^{\text {th }}$ century, becoming the third element (joining family and school) of the basic socializing tripod of contemporary societies. However, a fourth agent could be added to this tripod: peer group. The influence of ICTs on children and adolescents is usually mediated by relationships with peers. Relational perspective centered approaches to socializing processes show that relationship between parents and their children are more reciprocal and more dynamic than traditional approaches establish. However, although parents and children talk more openly, more frequently and also discuss about family decisions, conversations about ICTs not seem to be a preference. Adults tend to not accept they are less competent than children or adolescents in the use of audiovisual media, so they usually avoid communication on it or devaluate children competence, leading children to be more satisfied when talk to peers about ICTs than when they talk to adults.

The main aim of this research is to explore changes in relationships of 12-to-16 year old adolescents and their parents in a 5-years period.

\section{Method and procedure}

Article presents data from 2 samples of Catalan 12-to-16 year old boys and girls and their parents, obtained in 2003 and 2008, through a questionnaire.

This questionnaire was administrated in the high school classroom to adolescents after maintain several meetings with direction and parents association. Adolescents were asked to take home an envelope including a letter and a questionnaire to parents, which was returned to school after being fulfilled. Adolescents and their parents' questionnaires had codes in order to be matched. 


\section{Results}

Adolescents' answers show that the availability of computer, Internet connection, console and mobile phone is higher in 2008, while having own TV was more frequent in 2003. An increase in the frequency of conversations about ICTs is also observed, in all cases excepting teachers. In general terms, and regarding satisfaction with this communication maintained with adults (father, mother and teachers), in 2003 adolescents were significantly more satisfied with conversations than in 2008. However, when conversations are maintained with friends, satisfaction depends on the media, being in 2003 more satisfactory those conversations related to TV and in 2008 those related to Internet and computer. Exploring the relation between frequency of communication and satisfaction with it, in 2008 a positive correlation is observed when it is referred to TV and computers and maintained with father or mother. Whereas in 2003 correlations are negative if they are about Internet with father or videogames with mother, and positive if they are on TV with mother. Frequency of conversations about TV, computer or Internet with same age friends are positively correlated with satisfaction that produce in both 2003 and 2008 samples, and also if they are with older friends in 2008. No relation is observed when communication is with a teacher.

Parents' answers about the satisfaction they think their child has when maintain a conversation about media, show that attributions made by parents in 2003 were almost with no exception more satisfactory than in 2008.

Comparing adolescents' and their parents' answers we observe that parents attribute more satisfaction than the own adolescent/child reports on the conversations with father about the computer, and coincide when conversations are related to the other explored ICTs, in both samples. Also a positive correlation between adolescents' satisfaction and parents' attribution, is observed related to all the ICTs. When communication is with mother there are no significant differences between answers given by adolescents and parents' attributions in any case. Positive correlations are found when conversations are related to $\mathrm{TV}$ and videogames, in both samples, and to Internet in 2003. Parents attributed more satisfaction than own adolescents felt to the conversations maintained with a teacher related to all the studied ICTs in 2003, and to computer and the Internet in 2008. Positive correlation is only observed in answers related to TV. In 2008, communication with friends about TV was more satisfactory to adolescent than their parents thought and coincident about the other ICTs, showing a positive correlation in all cases; whereas in 2003 attribution of parents is higher than the adolescent satisfaction in conversations about computer and the Internet with friends.

\section{Discussion}

Adolescents and their parents talk little about ICTs and these conversations aren't satisfactory. It is also observed that during a period of five years frequency has increased and satisfaction has decreased in Catalonia. It seems that parents assume the need of an intergenerational approach and consider they must be more involved in ICTs as a topic in which their children are interested in. However, the more frequent conversations are the more adolescents realize that their parents do not know deeply what they are talking about, causing dissatisfaction with this communication. In addition, parents have perceptual biases about their child's satisfaction with it. Consequently, both parents and adolescents should contribute with their best in order to diminish the distance between generations when talking about ICTs.

Positive correlation between frequency and satisfaction with conversations with both father and mother in 2008 show a change in parents' attitude, being more positive about the possibilities that ICTs have in the development of children and adolescents.

Changes in relationships with friends are also observed related to having a more homogeneous perception of peer group, being the experience in the use of technologies a decisive factor of satisfaction with communication with friends. 


\section{Agradecimientos}

El presente artículo se ha desarrollado en el marco de los proyectos: La influencia de las tecnologías de la información y la comunicación en la vida de chicos/as y adolescentes, y estudio de las interacciones y la comunicación que mantienen con los adultos, con número de referencia $\mathrm{BSO} 2002$ 03180, financiado por el Ministerio de Ciencia y Tecnología; y El bienestar psicológico y los valores aspirados en diferentes grupos de población adolescente y adulta: relación con el uso del tiempo libre y con el uso de las NTICs, con número de referencia SEJ2007-62813/PSIC, financiado por el Ministerio de Ciencia e Innovación.

\section{Referencias}

Alsinet, C., Pérez, R. M. y Agulló, M. J. (2003). Adolescentes y percepciones del riesgo. JOVENes, Revista de Estudios sobre Juventud, 18, 90-101.

Bressand, A. y Distler, C. (1995). La planète relationnelle. París: Flammarion. (Trad. castellano: $E l$ planeta relacional. Barcelona: Proa, 1997).

Cantero, P. y Gordillo, P. (1999). Juventud y entorno familiar. Madrid: Injuve.

Casas, F. (1998). Infancia: perspectivas psicosociales. Barcelona: Paidós.

Casas, F. (2001). Video games: between parents and children. En I. Hutchby y J. Moran-Ellis (Eds.). Children, technology and culture. The impacts of technologies in children's everyday lives (pp. 4257). Londres: Routledge/Falmer.

Casas, F. (2006). Infancia y representaciones sociales. Política y Sociedad, 43, 27-42.

Casas, F., Alsinet, C., Pérez Tornero, J. M., Figuer, C., Leiva, E., González, M. y Pascual, S. (2000). Las tecnologías de la información y la comunicación entre los padres y los hijos. Intervención Psicosocial, 9, 335-360.

Casas, F., Figuer, C., González, M., Malo, S. y Bertran, I. (2005). Medios audiovisuales y culturas adolescentes: nuevos usos, satisfacciones y diferencias de género. En J. M. Sabucedo, J. Romay y A. López-Cortón (eds.). Psicología social y problemas sociales. Psicología política, inmigración y comunicación social. Volumen II (pp. 295-300). Madrid: Biblioteca Nueva, S. L.

Casas, F., González, M., Figuer, C. y Malo, S. (2007). The penetration of audio-visual media into adolescent cultures in Spain between 1999 and 2003. En F. Casas, I. Rizzini, R. September, P. E. Mjaavath y U. Nayar (eds.). Adolescents and audio-visual media in five countries (pp. 105125). Girona: Documenta Universitaria.

Casas, F., Mjaavatn, P.E., Nayar, U., Rizzini, I., September, R., Figuer, C., González, M. y Malo, S. (2007). Adolescents, their parents and audiovisual media: evaluations and interpersonal relationships in five countries. En F. Casas, I. Rizzini, R. September, P. E. Mjaavath y U. Nayar (eds.). Adolescents and audio-visual media in five countries (pp. 11-62). Girona: Documenta Universitaria.

Feixa, C. (2001). Generació @.La joventut del segle XXI. Barcelona: Observatori Català de la Joventut.

Harris, J. R. (1995). Where is the child's environment? A group socialization theory of development. Psychological Review, 102, 458-489.

Harris, J. R. (2006). Are peers more important than parents during the process of development? Yes. En A. M. Guest (Ed.). Taking sides: Clashing views in lifespan development. Dubuque, Iowa: McGraw-Hill/Dushkin.

Kuczynski, L. (2003). Beyond bi-directionality: bilateral conceptual frameworks for parentalchild relations. En L. Kuczynski (Ed.). Handbook of dynamics in parent-child relations (pp. 3-24). Twin Oaks, CA: Sage.

Lollis, S. y Kuczynski, L. (1997). Beyond one hand clapping: seeing bidireccionality in parent-child relations. Journal of Social and Personal Relationships, 14, 441-461.

Maccoby, E. y Martin, J. (1983). Socialization in the context of the family: parent-child interaction. En E.M. Hetherington y P.H. Mussen (Eds.). Handbook of child psychology: vol. 4. Socialization, personality, and social development (pp. 1-101). Nova York: Wiley.

Malo, S. (2009). Culturas mediáticas adolescentes: Un estudio psicosocial centrado en el teléfono 
móvil. Servidor de Tesis Doctorales en Red (TDX). http://www.tdx.cat/TDX-0223109134709. Depósito Legal: GI-302-2009

Reiss, H.G.T., Collins, W.A. y Berscheild, E. (2000). The relationship context of human behavior and development. Psychological Bulletin, 126, 844-872.

Suess, D., Suoninen, A., Garitaonandia, C., Juaristi, P., Koikkalainen, R. y Oleaga, J. A. (1998). Media use and the relationships of children and teenagers with their peer groups. European Journal of Communication, 13, 521-538.

Suoninen, A. (2001). The role of media in peer group relations. En S. Livingstone y M. Bovill (Eds.). Children and their changing media environment: a european comparative study (pp. 201-219). Londres: Lawrence Erlbaum .

Tapscott, D. (1998). Growing up digital: the rise of the net generation. Nueva York: McGraw Hill.

Manuscrito recibido: 18/01/2010

Revisión recibida: 29/01/2010

Manuscrito aceptado: 04/02/2010 